\title{
miR-146a negatively regulates NK cell functions via STAT1 signaling
}

\author{
Dongqing Xu, Qiuju Han, Zhaohua Hou, Cai Zhang and Jian Zhang
}

It is known that natural killer (NK) cell function is downregulated in chronic hepatitis B (CHB)-infected patients and in hepatic carcinoma (HCC) patients, but the mechanisms underlying this functional downregulation are largely unclear. In this study, microRNA (miR)-146a expression increased in NK cells from CHB and HCC patients compared with NK cells from healthy donors, and miR-146a levels were negatively correlated to NK cell functions. Overexpression of miR-146a reduced NK cell-mediated cytotoxicity and the production of interferon (IFN)- $\gamma$ and tumor necrosis factor- $\alpha$, which were reversed upon inhibition of miR-146a. In NK cells, miR-146a expression was induced by interleukin (IL)-10 and transforming growth factor- $\beta$, but reduced after treatment with interleukin-12, IFN- $\alpha$ and IFN- $\beta$. We further revealed that miR-146a regulated NK cell functions by targeting STAT1. Taken together, upregulated miR-146a expression, at least partially, attributes to NK cell dysfunction in CHB and HCC patients. Therefore, miR-146a may become a therapeutic target with great potential to ameliorate NK cell functions in liver disease. Cellular \& Molecular Immunology (2017) 14, 712-720; doi:10.1038/cmi.2015.113; published online 21 March 2016

Keywords: CHB; HCC; miR-146a; natural killer cells; STAT1

\section{INTRODUCTION}

Natural killer (NK) cells are innate immune cells that are important for the host defense against viruses and transformed cells. NK cells mediate several functions including cytotoxicity and the production of cytokines and chemokines. Many factors can affect the function of NK cells. On the one hand, NK cells express both activating and inhibitory receptors; thus, the balance between positive signals and negative signals determines the functional outcome of NK cells. ${ }^{1}$ On the other hand, NK cell function can be modulated by various cytokines, including stimulatory cytokines interleukin (IL)-12 and interferon (IFN)- $\alpha,{ }^{2,3}$ and inhibitory cytokines interleukin (IL)-21 and transforming growth factor (TGF) $-\beta .{ }^{4}$ However, the understanding of the intracellular molecular mechanism and programs that define NK cell function is incomplete.

Transcription factors such as myeloid elf-1-like factor (MEF) and microphthalmia-associated transcription factor (MITF) have been identified as contributors to NK cells programming at the transcription level. In addition, effector molecules, such as IFN- $\gamma$, perforin and granzymes, which are important for NK cell functions, are partly controlled at the posttranscriptional level. ${ }^{5}$ Recently, microRNAs (miRNAs) have drawn increasing interest and have become better understood as a posttranscriptional regulatory mechanism for effector molecules of NK cells. miRNAs are small non-coding RNAs, which regulate gene expression by inducing translational repression, cleavage or destabilization of the target messenger RNAs (mRNAs). The importance of miRNAs for NK cell function was confirmed by disrupting global miRNAs in mouse NK cells. ${ }^{6}$ Three research groups have identified the top 20 miRNAs expressed in mouse and human NK cells using next-generation sequencing. ${ }^{7-9}$ Thus far, the miRNAs that have been identified as ones involved in NK cell development are miR-150 and miR-181a/b, which target $\mathrm{c}-\mathrm{Myb}^{10}$ and NEMO-like kinase ${ }^{11}$, respectively. Moreover, miRNAs have been shown to regulate NK cell functions. For instance, several miRNAs regulate IFN- $\gamma$ production, a key cytokine of NK cells that is involved in a multilayered regulatory network. IFN- $\gamma$ production is suppressed by miR-29 and miR-15a/15b/16, members of the miR-15/16 family, which target the $3^{\prime}$-untranslated region (UTR) of IFN- $\gamma$ in NK cells. ${ }^{12,13}$ In contrast, miR-155 promotes IFN- $\gamma$ production by targeting SHP-1, the upstream gene of IFN $-\gamma^{14}$ Furthermore, some miRNAs, such as miR-27a-5p, miR-378

Institute of Immunopharmaceutical Sciences, School of Pharmaceutical Sciences, Shandong University, Jinan 250012, China

Correspondence: J Zhang, Institute of Immunopharmaceutical Sciences, School of Pharmaceutical Sciences, Shandong University, 44 Wenhua West Road, Jinan 250012, China.

E-mail: zhangj65@sdu.edu.cn

Received: 21 June 2015; Revised: 10 December 2015; Accepted: 21 December 2015 
and miR-30e, perturb NK cell cytolytic activity by targeting the granule proteins granzyme $\mathrm{B}$ and perforin.,15 Finally, miR-30c-1-3p enhances NK cell cytotoxicity by targeting HMBOX1, an inhibitory transcription factor for cytokines. ${ }^{16}$ In summary, miRNAs can modulate both the development and function of NK cells.

Both miR-146a and miR-146b belong to the miR-146 family, and they are located on human chromosomes 5 and 10, respectively. Functionally important, miR-146a not only exerts a key role in tumor progression and viral infections but it is also involved in innate and adaptive immune responses. Elevated miR-146 expression levels, which are likely to reflect the infiltration of inflammatory cells and increased levels of mediators tumor necrosis factor (TNF)- $\alpha$ and IL-1 $\beta$, were found in tissues related to chronic inflammatory diseases such as psoriasis and rheumatoid arthritis. ${ }^{17}$ In addition, miR-146a is an important modulator of innate immune cell differentiation and function, as well as adaptive immunity. In monocytes, miR-146a attenuates TLR4 signaling and contributes to endotoxin tolerance by targeting TRAF6 and IRAK1. ${ }^{18,19}$ In macrophages, miR-146a feedback inhibits RIG-I-dependent type I IFN production by targeting TRAF6 and IRAK1. Furthermore, in chronic hepatitis $\mathrm{B}(\mathrm{CHB})$ patients, miR-146a depresses T-cell immune function by targeting STAT1. ${ }^{20}$ Although miR-146a is 1 of the top 20 miRNAs in NK cells, ${ }^{9}$ its mechanisms of action on NK cell functions remain unclear.

In this study, we found that the cytotoxicity of NK cells from the peripheral blood of $\mathrm{CHB}$ and HCC patients negatively correlated with miR-146a expression levels. Further investigation showed that miR-146a regulated NK cell function by targeting STAT1, a transcription factor critical for NK cell function. Finally, we found that the expression of miR-146a in NK cells was differentially regulated by specific cytokines. Therefore, manipulating miR-146a expression maybe a new way of rescuing NK cell function.

\section{MATERIALS AND METHODS}

\section{Study subjects}

Peripheral blood samples of $\mathrm{CHB}$ and HCC patients were obtained from Qilu Hospital, with informed consent from all participants, in accordance with the Ethics Committee of Shandong University. The clinical data for these patients are shown in Table 1 . There were 62 HCC patients, including 38

Table 1 Clinical features of CHB and HCC patients and healthy donors included in the study

\begin{tabular}{lccc}
\hline & $H D$ & $C H B$ & $H C C$ \\
\hline Number & 62 & 70 & 62 \\
Gender (M/F) & $14 / 48$ & $23 / 47$ & $7 / 55$ \\
Age (years) & $50.56 \pm 13.88$ & $48.18 \pm 13.42$ & $58.49 \pm 8.283$ \\
HBsAg (Pos/Neg) & $0 / 62$ & $70 / 0$ & $38 / 24$ \\
HBeAg (Pos/Neg) & $0 / 62$ & $41 / 29$ & $25 / 37$ \\
\hline
\end{tabular}

Abbreviations: $\mathrm{CHB}$, chronic hepatitis B; F, female; HCC, hepatic carcinoma; $\mathrm{HD}$, healthy donor; $\mathrm{M}$, male; Neg, negative; Pos, positive. patients infected with hepatitis B virus. None of the healthy donors (HDs) had a history of hepatic disease.

\section{Cell culture and lentiviral transduction}

The human hepatocellular carcinoma cell line HepG2 was maintained in Dulbecco's modified Eagle's medium (Gibco/ BRL, Grand Island, NY, USA) supplemented with $10 \%$ fetal bovine serum (FBS). The human NK cell line NK-92 was purchased from American Type Culture Collection (Manassas, VA, USA) (CRL-2407) and maintained in $\alpha$-minimum essential medium (Gibco/BRL) supplemented with $12.5 \%$ horse serum, $12.5 \%$ FBS, $100 \mathrm{U} / \mathrm{ml}$ recombinant human IL (rhIL)-2, $0.1 \mathrm{mM} \beta$-mercaptoethanol and $0.02 \mathrm{mM}$ folic acid. All cells were incubated at $37^{\circ} \mathrm{C}$ and $5 \% \mathrm{CO}_{2}$, and were used $<6$ months after they were initially thawed. Primary NK cells were isolated from donor peripheral blood using an NK cell isolation kit (Miltenyi Biotech $\mathrm{GmbH}$, Bergisch Gladbach, Germany) and cultured in RPMI-1640 medium (Gibco/BRL) supplemented with $10 \%$ FBS and $100 \mathrm{U} / \mathrm{ml}$ rhIL-2.

The miR-146a overexpression lentivirus, miR-146a inhibition lentivirus and hsa-STAT1-ORF lentivirus (3'-UTR less) were purchased from GenePharma (Shanghai, China). Viral titer was performed at 100:1 for NK cells transduction with $4 \mu \mathrm{g} / \mathrm{ml}$ polybrene. In order to establish miR-146aoverexpressing or miR-146a-inhibited NK92 cell lines, NK-92 cells were selected with puromycin $(1 \mu \mathrm{g}$; Sigma-Aldrich, St Louis, MO, USA) for 1 week.

\section{RNA isolation and quantitative real-time PCR}

Total RNA was isolated using TRIzol reagent (Invitrogen, Carlsbad, CA, USA) according to the manufacturer's instructions. Complementary DNA was synthesized using M-MLV reverse transcriptase (Invitrogen). The expression level of miR-146a was determined by quantitative PCR (qPCR) using a SYBR Green Real-Time PCR Kit (Roche, Mannheim, Germany) on an iQ5 Real-Time PCR detection system (Bio-Rad, Hercules, CA, USA). The primers for miR-146a-5p were purchased from RiboBio (Guangzhou, China). The relative expression of the miRNA was calculated using the $2^{-\Delta \Delta \mathrm{Ct}}$ method.

\section{Cytotoxicity assay}

NK-92 cell-mediated cytotoxicity was determined via flow cytometry using the CFSE/7AAD cytotoxicity assay. HepG2 cells were used as target cells. NK-92 cells were labeled with $200 \mathrm{nM}$ CFSE (Beyotime, Nanjing, China) for $15 \mathrm{~min}$ at $37^{\circ} \mathrm{C}$ in a volume of $2 \mathrm{ml}$. After $15 \mathrm{~min}$, these cells were washed twice with phosphate-buffered saline (PBS) and immediately used as effector cells in the cytotoxicity assay at effector: target (E:T) ratios of 10:1, 5:1 and 2.5:1. HepG2 cells incubated alone were used to measure basal cell death. Six hours post incubation in complete medium, cell mixtures were washed twice with PBS and incubated for $15 \mathrm{~min}$ at room temperature in buffer with $20 \mu \mathrm{g} / \mathrm{ml}$ of 7-amino actinomycin D (KeyGEN BioTECH, Nanjing, China). Acquisition was performed using 
the FACSCalibur system (BD Biosciences, San Jose, CA, USA). Specific lysis was calculated as followed: $\%$ ratio $=\left(\mathrm{CFSE}^{-}\right.$ $\left.7 \mathrm{AAD}^{+}\right) / \mathrm{CFSE}^{-} \times 100 \%, \%$ specific lysis $=\%$ ratio of sample $\%$ ratio of basal.

The cytotoxicity of primary NK cells was determined using an lactate dehydrogenase (LDH) assay (Beyotime) performed according to the manufacturer's instructions. Briefly, HepG2 cells were seeded onto 96-well plates at a density of $1 \times 10^{4}$ cells per well, and primary NK cells were added to each well at an E:T ratio of 10:1. After $12 \mathrm{~h}$, the cells were gently centrifuged at $200 \mathrm{~g}$ for $5 \mathrm{~min}$, and $100 \mu \mathrm{l}$ of supernatant was transferred to a new 96-well microplate. The maximum amount of LDH release (high control) was determined by lysing cells with a final concentration of $1 \%$ Triton X-100. The supernatants of untreated HepG2 cells (which spontaneously release LDH) were used as a low control. To each well containing supernatant, $100 \mu \mathrm{l}$ of the detection substrate, a tetrazolium salt, was added, and the resulting mixture was incubated in the dark for $30 \mathrm{~min}$. Absorbance was measured at $490 \mathrm{~nm}$ using a reference wavelength of $630 \mathrm{~nm}$. After subtracting out the low control values, the percent cytotoxicity was calculated in relation to the high control values.

\section{Flow cytometry}

Cells were collected, washed twice with PBS and incubated with antibodies for $45 \mathrm{~min}$ at $4{ }^{\circ} \mathrm{C}$. For detection of intracellular cytokines, cells were fixed and permeabilized, and stained with a saturating amount of antibodies for $1 \mathrm{~h}$ at $4{ }^{\circ} \mathrm{C}$. All stained cells were measured using a FACSCalibur flow cytometer (BD Biosciences), and data were analyzed using FCS Express software (De Novo Software, Glendale, CA, USA). The following antibodies were used in this study: anti-granzyme $\mathrm{B}$, anti-perforin, anti-IFN-gamma, anti-TNF-alpha, anti-NKG2D, anti-NKG2A, anti-NKp30, anti-NKp44, anti-NKp80 and anti-CD107a purchased from BD Biosciences, BioLegend (San Diego, CA, USA) or eBioscience (San Diego, CA, USA).

\section{Western blot analysis}

Cells were collected, solubilized in lysis buffer and incubated on ice for $30 \mathrm{~min}$. Sodium dodecyl sulfate-polyacrylamide gel electrophoresis was performed according to standard protocols. After electrophoresis, proteins were transferred to polyvinylidene difluoride membranes (Millipore, Billerica, MA, USA). The membranes were blotted with antibodies for $12 \mathrm{~h}$ at $4{ }^{\circ} \mathrm{C}$ followed by blocking in 5\% nonfat milk. Horseradish peroxidase-conjugated secondary antibodies (Genetech, Shanghai, China) were used in conjunction with an enhanced chemiluminescence system (Millipore) to detect protein expression. The following antibodies were used: rabbit polyclonal anti-STAT1 and rabbit polyclonal anti-STAT1 (phosphor-Tyr701) purchased from Biobasic (Markham, Ontario, Canada); rabbit polyclonal anti-IRAK-1 and rabbit polyclonal anti-TRAF6 obtained from Santa Cruz Biotechnology (Santa Cruz, Dallas, TX, USA).

\section{Cytokines}

Recombinant human TGF- $\beta 1$, IL-10 and IL-6 were purchased from PeproTech (Rocky Hill, NJ, USA). IFN- $\alpha$ and IFN- $\beta$ were purchased from Changsheng Life Sciences (Changchun, China).

\section{Statistical analysis}

All data are presented as the means \pm s.d. of three or more independent experiments. Statistical analysis was performed using a paired Student's $t$-test. Statistically significant differences were set at ${ }^{\star} P<0.05,{ }^{*} P<0.01$.

\section{RESULTS}

NK cell cytotoxicity activity is negatively associated with miR-146a expression levels

As stated in previous reports, NK cells isolated from $\mathrm{CHB}$ and HCC patients displayed decreased cytolytic activity against HepG2 cells compared with NK cells from HDs (Figure 1a). To evaluate the effect of miR-146a in NK cells, the expression level of miR-146a in NK cells was measured by qPCR. Compared with HDs, miR-146a levels in NK cells increased by 2.5 -fold in both $\mathrm{CHB}$ patients $(P<0.05)$ and HCC patients $(P<0.01$; Figure $1 \mathrm{~b})$. Further analysis showed that the expression level of miR-146a was negatively related to NK cellmediated cytotoxicity (Figure 1c). In addition, miR-146a expression in primary NK cells from HDs increased when cultured with serum from CHB and HCC patients (Figure 1d). These results suggest that miR-146a might act as a negative regulatory molecule for NK cell function.

\section{MiR-146a negatively regulates NK cell function}

To confirm the role of miR-146a in NK cell function, miR-146a-overexpressing (NK92-146a-over) and miR-146ainhibited (NK92-146a-inh) NK cells were generated using lentiviral transduction (Supplementary Figure 1). As shown in Figure 2a (left), the cytotoxicity of NK92-146a-over cells was significantly lower than the control cells (NK92-LV-NC) at different E:T ratios. In contrast, the cytotoxic activity of NK92146a-inh was higher than NK92-LV-NC cells (Figure 2a, right). Furthermore, overexpression of miR-146a significantly inhibited the production of the effector molecules IFN- $\gamma$, TNF- $\alpha$ and perforin (Figure 2b), whereas inhibition of miR-146a promoted cytokine production including that of TNF- $\alpha$ and IFN- $\gamma$ (Figure 2c). Similarly, the cytotoxic activity of primary NK cells from HDs was inhibited by miR-146a overexpression and enhanced by miR-146a inhibitor treatment (Figure 2d). Of note, miR-146a inhibition improved the cytotoxic activity of NK cells from $\mathrm{CHB}$ and HCC patients (Figure 2e). However, overexpression of miR-146a did not influence the expression of NK cell receptors significantly (Supplementary Figure 1E). These results indicate that miR-146a was functional in NK cells and displayed negative effects on the cytotoxic activity of NK cells. 


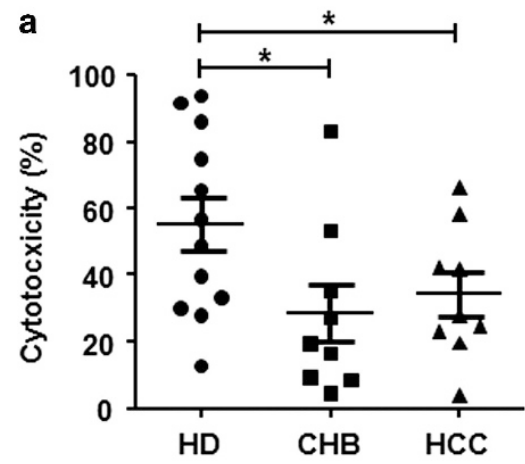

b
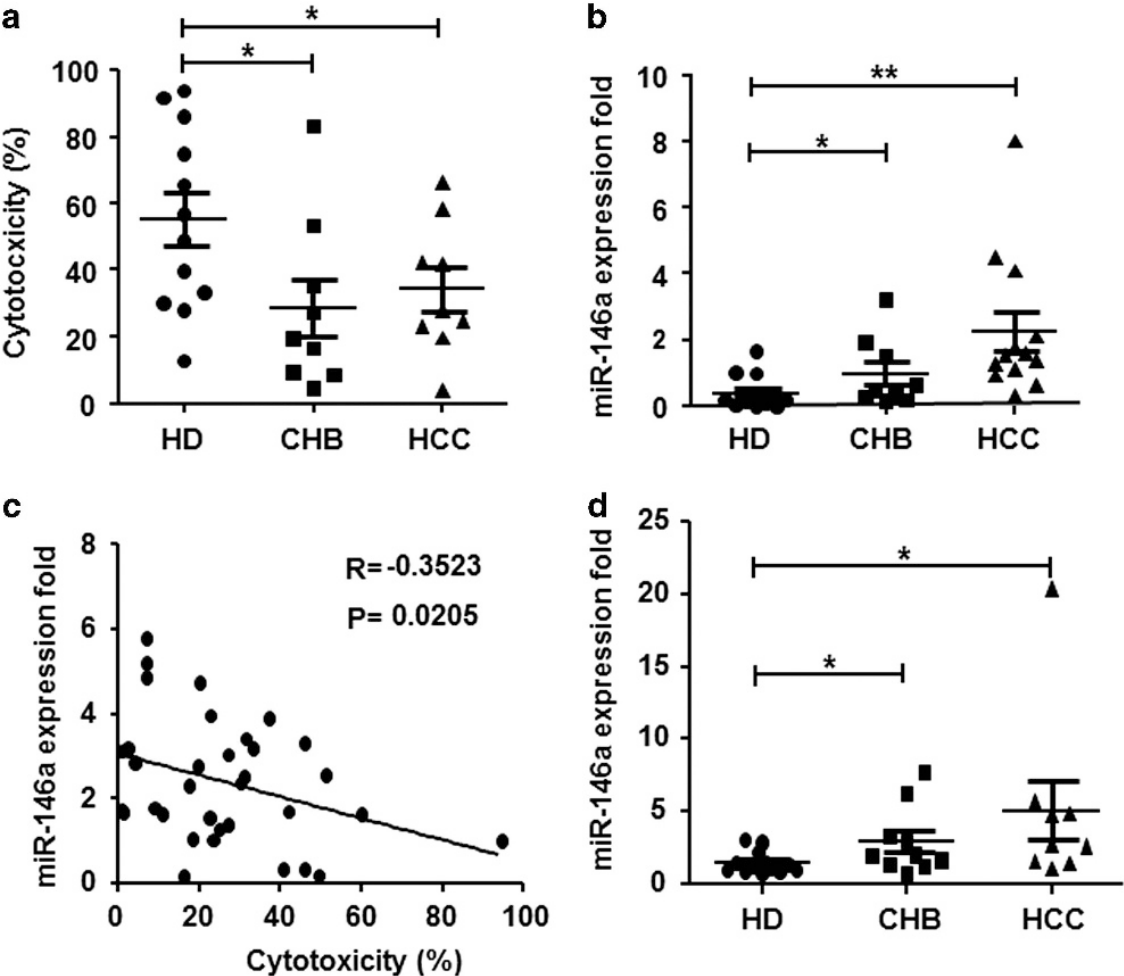

Figure $1 \mathrm{NK}$ cell cytotoxicity is negatively associated with miR-146a expression levels. NK cells were isolated from CHB and HCC patients using a MACS NK cell isolation kit. (a) The cytotoxicity of NK cells to HepG2 cells was detected using an LDH cytotoxicity assay. HD $(n=12)$, CHB $(n=9)$ and HCC $(n=9)$. (b) miR-146a expression levels were quantified by qPCR. The results were normalized to an endogenous control, RNU6B. HD $(n=12), \mathrm{CHB}(n=9)$ and HCC $(n=13)$. (c) The correlation between the cytotoxicity of NK cell and the expression level of miR-146a $(n=34)$. (d) Primary NK cells were treated with serum from CHB and HCC patients for $4 \mathrm{~h}$, and then the expression of miR-146a was quantified by qPCR. Statistical significance was determined as ${ }^{*} P<0.05$; ${ }^{* *} P<0.01$ compared with the control. CHB, chronic hepatitis B; HCC, hepatic carcinoma; HD, healthy donors; LDH, lactate dehydrogenase; miR, microRNA; NK, natural killer; qPCR, quantitative PCR.

miR-146a expression can be regulated by cytokines associated with NK cell activation

NK cell activation can be influenced by exposure to various cytokines. Therefore, we assessed whether NK-related cytokines could regulate the expression of miR-146a. Several studies have shown that the serum levels of TGF- $\beta 1$, IL- 6 and IL- 10 are increased in HCC and CHB patients. ${ }^{21,22}$ In this study, we found that in healthy NK cell donors, miR-146a expression was induced when the cells were treated with IL-10 $(30 \mathrm{ng} / \mathrm{ml})$ or TGF- $\beta 1$ ( $30 \mathrm{ng} / \mathrm{ml})$, whereas miR-146a expression did not change significantly with exposure to IL-6 (Figure 3a). On the contrary, stimulatory factors such as poly(I:C), IL-12 and IFN- $\alpha$ decreased miR-146a expression in NK cells (Figure 3b). These data suggest that cytokines known to suppress NK cell function could induce the expression of miR-146a, whereas cytokines known to stimulate NK cell activation could suppress the expression of miR-146a.

\section{MiR-146a targets STAT1 in NK cells}

To clarify the mechanism of action of miR-146a in NK cells, we investigated potential targets of miR-146a. Previously published research has found that miR-146a targets IRAK1 and TRAF6 in monocytes and macrophages, and has an inhibitory role in autoimmune disease. ${ }^{18,19}$ In our study, miR-146a did not significantly affect the protein expression of IRAK1 and TRAF6 (Figures 4a and b), suggesting that IRAK1 and TRAF6 may not be targets of miR-146a. STAT1, a key signaling protein, which contributes to the type I IFN pathway in lupus patients, was reported to be another important target of miR-146a. ${ }^{23}$ Moreover, by targeting STAT1, miR-146a could suppress T-cell function. ${ }^{20}$ In our study, we found a decreased amount of STAT1 in NK92146a-over cells (Figure 4a) and an increased amount of STAT1 in NK92-146a-inh cells (Figure 4b). In addition, the level of STAT1 in primary NK cells from HDs was reduced by miR-146a (Figure 4c). This is consistent with the lower levels of STAT1 measured in NK cells from CHB and HCC patients compared with those from HDs (Figure 4d). These data demonstrate that miR-146a targets STAT1 in NK cells.

STAT1 signaling contributes to NK cell cytotoxic activity STAT1 is a cytoplasmic transcription factor activated by multiple cytokines and growth factors. ${ }^{24}$ Mice deficient in STAT1 display impaired basal NK cell cytolytic activity 

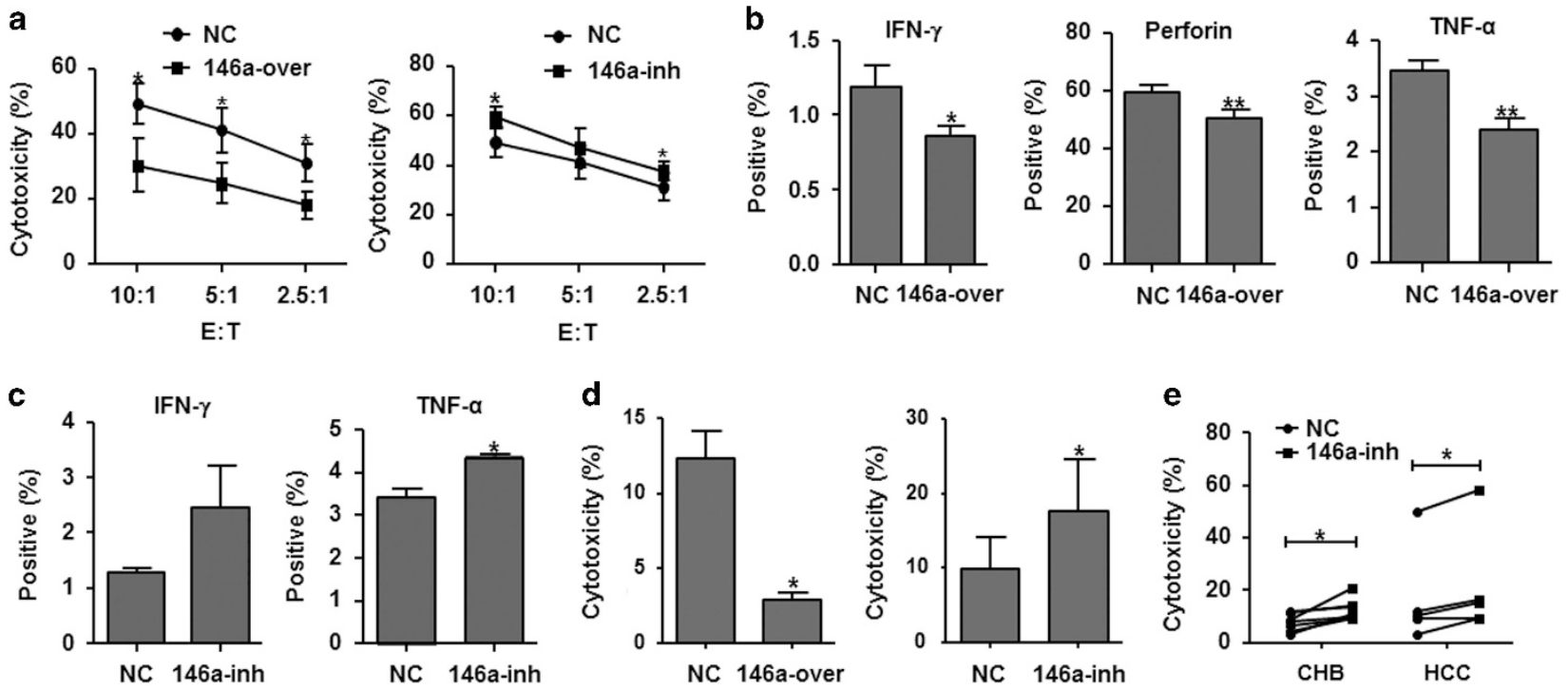

Figure 2 miR-146a negatively regulates NK cell function. The cytolytic activity of NK92-146a-over cells (left) and NK92-146a-inh cells (right) against HepG2 cells was analyzed using a CFSE/7AAD assay. (b, c) The protein levels of molecules associated with NK cell cytolysis were analyzed by flow cytometry. (d) Primary NK cells from healthy donors were transduced with miR-146a-overexpressing or miR-146ainhibiting lentivirus; $24 \mathrm{~h}$ later, the cytotoxicity of these NK cells against HepG2 cells was analyzed using an LDH cytotoxicity assay. (e) NK cells from $\mathrm{CHB}(n=6)$ and $\mathrm{HCC}(n=5)$ patients were transduced with miR-146a inhibitor lentivirus, and the cytotoxicity was detected using an LDH cytotoxicity assay at $E: T=10: 1$. Data are representative of three independent experiments, statistical significance was determined as ${ }^{*} P<0.05$ and ${ }^{*} P<0.01$ compared with the control. $\mathrm{CHB}$, chronic hepatitis B; HCC, hepatic carcinoma; LDH, lactate dehydrogenase; miR, microRNA; NK, natural killer.
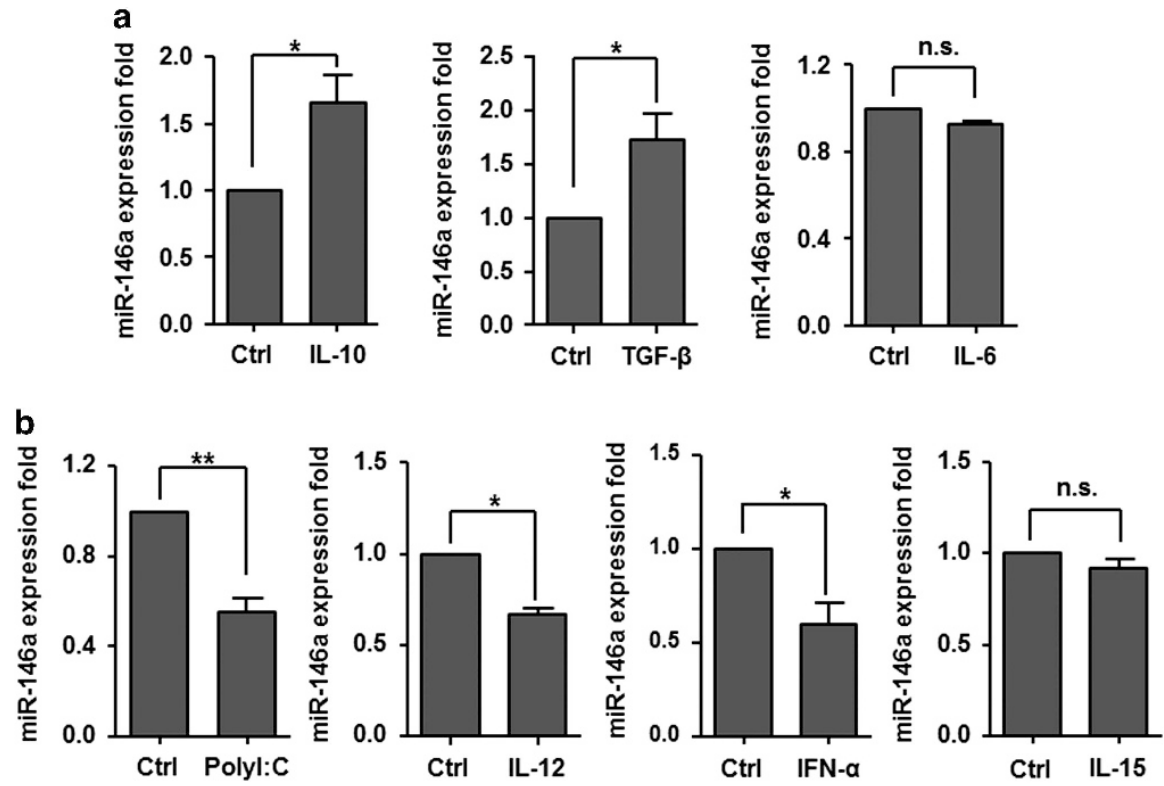

Figure 3 miR-146a expression is regulated by cytokines associated with NK cell activation. (a) Primary NK cells were treated with IL-10 (30 ng/ml), TGF- $\beta(30 \mathrm{ng} / \mathrm{ml})$ and IL-6 (30 ng/ml). ${ }^{33}$ After $4 \mathrm{~h}$, miR-146a expression was quantified by qPCR. (b) Primary NK cells were stimulated with poly(I:C) $(500 \mathrm{IU} / \mathrm{ml}), \mathrm{IL}-12(20 \mathrm{ng} / \mathrm{ml}), \mathrm{IL}-15(20 \mathrm{ng} / \mathrm{ml})$ and IFN- $\alpha(1,000 \mathrm{IU} / \mathrm{ml}){ }^{8,24,34}$ After $12 \mathrm{~h}$, miR-146a expression was quantified by qPCR. Data are representative of three independent experiments, statistical significance was determined as ${ }^{*} P<0.05$ and $* * P<0.01$ compared with the control. Ctrl, control; IL, interleukin; INF, interferon; miR, microRNA; NS, nonsignificant; NK, natural killer; TGF, transforming growth factor; qPCR, quantitative PCR.

in vitro. ${ }^{25}$ As shown in Figure 5a, IFN- $\alpha$ could increase NK activity, whereas an inhibitor of STAT1, fludarabine (Flu), decreased the cytolytic activity of NK cells, which could be reversed by the addition of IFN- $\alpha$ (Figure $5 \mathrm{a}$ ). Furthermore,
miR-146a overexpression-induced inhibition of NK cell cytotoxicity could be aggravated by fludarabine, which was similar to what was observed in fludarabine-treated NK cells (Figure 5b). Similar results were observed in primary NK cells 

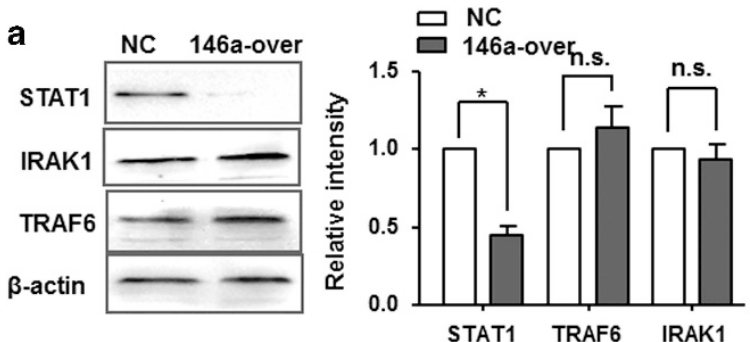

c
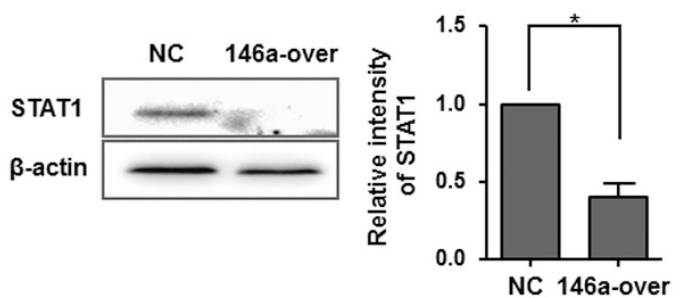

b
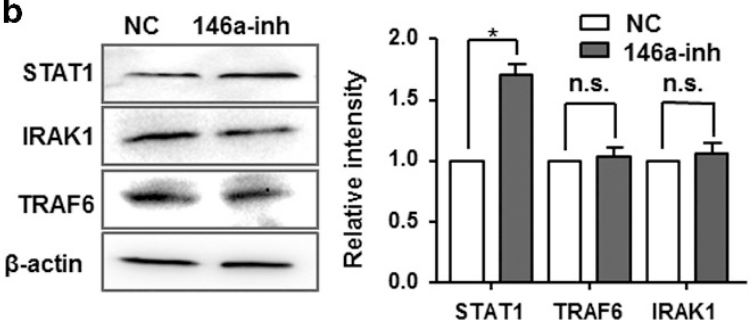

d
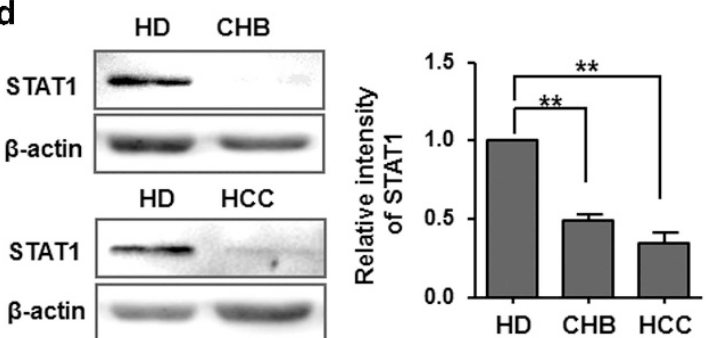

Figure 4 miR-146a targets STAT1 in NK cells. The protein expression of IRAK1, TRAF6 and STAT1 in (a) miR-146a-overexpressing NK92 cells and (b) miR-146a-inhibited NK-92 cells was measured by western blot. (c) Primary NK cells were transduced with miR-146a overexpression lentivirus; $24 \mathrm{~h}$ later, the expression of STAT1 was detected by western blot. (d) STAT1 expression of the mixed NK cells from $\mathrm{CHB}$ patients $(n=8)$ or HCC patients $(n=8)$ was detected. Data are representative of three independent experiments, statistical significance was determined as ${ }^{*} P<0.05$ and ${ }^{*} P<0.01$ compared with the control. CHB, chronic hepatitis B; HCC, hepatic carcinoma; miR, microRNA; NS, nonsignificant; NK, natural killer.
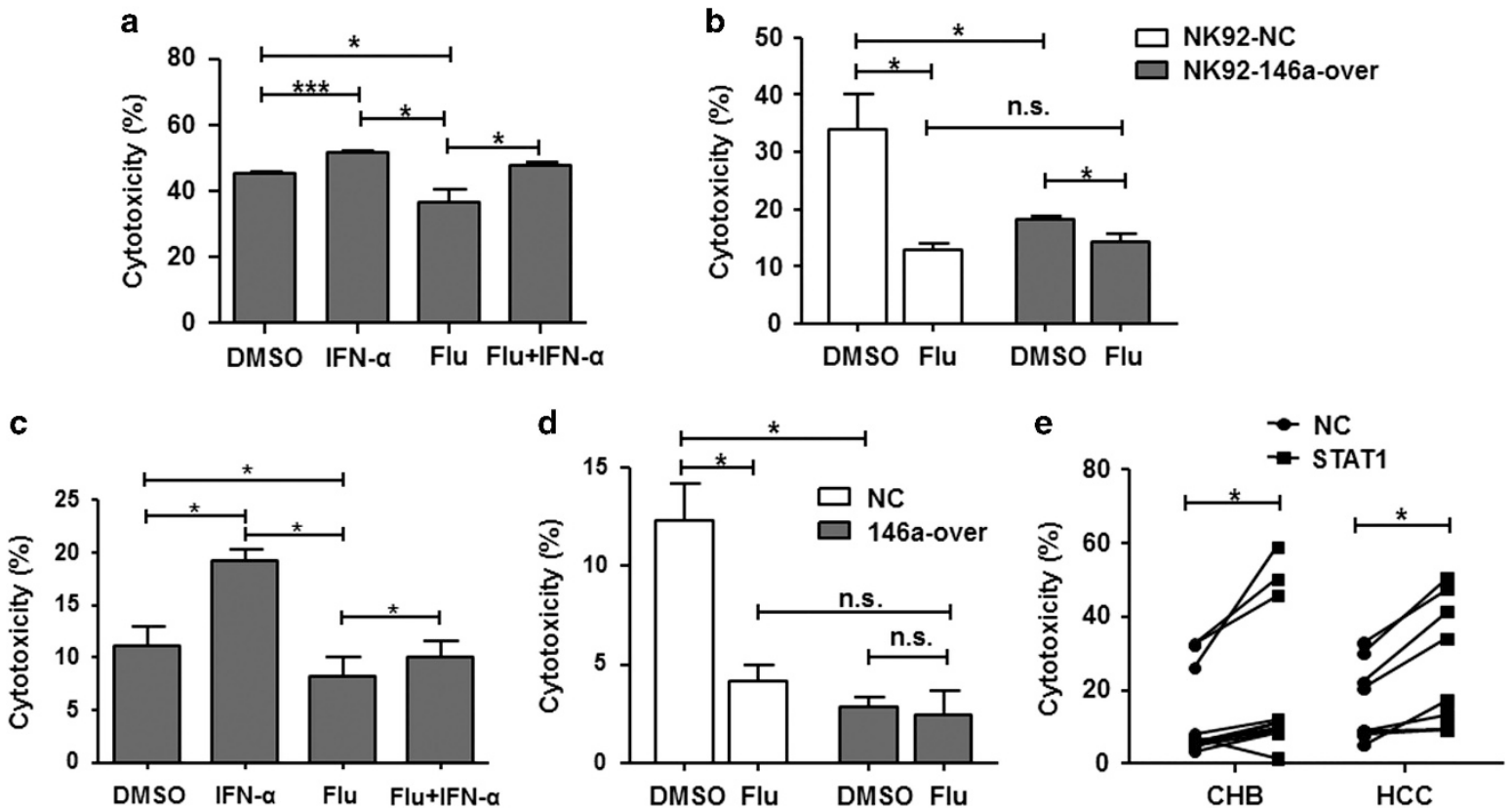

Figure 5 STAT1 signaling contributes to NK cell cytotoxic activity. NK-92 cells (a) or primary NK cells (c) were treated with IFN- $\alpha$ (1000 IU/ml), fludarabine ( $50 \mu \mathrm{M}$; Flu) or both for $24 \mathrm{~h}$; then, the cytotoxicity of these NK cells against HepG2 cells was analyzed. NK92146a-over cells (b) or primary NK cells transduced with miR-146a overexpression lentivirus for $24 \mathrm{~h}$ (d) were treated with or without fludarabine, and then the cytotoxicity was detected. (e) NK cells from patients of CHB $(n=10)$ and HCC ( $n=8)$ were transduced with hasSTAT1-ORF lentivirus; $24 \mathrm{~h}$ later, the cytotoxicity was detected. Data are representative of three independent experiments, statistical significance was determined as ${ }^{*} P<0.05$ and ${ }^{* *} P<0.001$. CHB, chronic hepatitis $\mathrm{B}$; HCC, hepatic carcinoma; INF, interferon; miR, microRNA; NK, natural killer. 
from HDs (Figures $5 \mathrm{c}$ and d). In addition, overexpression of STAT1 with hsa-STAT1-ORF lentivirus (3'-UTR less) rescued the decreased cytotoxic activity of NK cells from $\mathrm{CHB}$ and HCC patients (Figure 5e). These results indicated that the STAT1 signaling pathway contributes to NK cell function, and that miR-146a negatively regulates NK cell function by interfering with STAT1 expression.

miR-146a expression in NK cells from HCC or CHB patients can be reversed by cytokine-based therapy in vitro

Various cytokines have been evaluated as potential anticancer drugs. IFN- $\alpha$ is used for the treatment of cancers as well as HBV and HCV infections, ${ }^{26,27}$ IL-12 treatment maybe highly beneficial in the context of therapies by enhancing NK cell functions in cancer patients. ${ }^{28}$ To evaluate whether these cytokines could reverse miR-146a expression in NK cells from $\mathrm{CHB}$ and HCC patients, isolated NK cells were treated with IFN- $\alpha / \beta$ and IL-12. As shown in Figure $6 \mathrm{a}, \mathrm{IL}-12$ and IFN- $\alpha$ treatment decreased the expression of miR-146a, particularly for patients with high baseline levels of miR-146a. Meanwhile, the expression of the functional marker CD107a was induced (Figure 6b), indicating the functional amelioration of NK cells. These findings indicated an underlying mechanism of cytokine therapy and that miR-146a might be a marker for NK cell dysfunction.

\section{DISCUSSION}

As the initial discovery of miRNA in 1993, hundreds of miRNAs in mammals have been identified. A range of them have been found to be involved in physiological processes and disease. Human diseases, including those of the lung, liver and kidney, as well as infectious diseases are associated with dysregulated miRNA expression. In the immune system, miRNAs participate in the differentiation and development of immune cells, as well as the innate and acquired immune responses. Among the miRNAs discovered, the roles of miR-146a as a negative regulator in monocyte, macrophages and $\mathrm{T}$ cells have been addressed. ${ }^{19,20,29}$ However, the role of miR-146a in NK cells is still unclear.

In our study, we discovered that NK cells isolated from $\mathrm{CHB}$ and HCC patients had higher expression levels of miR-146a that were accompanied by a suppression of NK cell cytotoxic activity. Further analysis showed that NK cell-mediated cytolysis was negatively associated with the expression levels of miR-146a (Figure 1). Using lentiviral constructs to suppress or overexpress miR-146a, we demonstrated that the overexpression of miR-146a reduced NK cell cytolysis activity and cytokine production, acting as a negative regulator in NK cells. Importantly, the suppression of miR-146a improved the cytolytic ability of NK cells from CHB and HCC patients (Figure 2). These findings indicate that high expression levels of miR-146a might be involved in the dysfunction of NK cells in $\mathrm{CHB}$ and HCC patients, although what induces the increased miR-146a in NK cells remains unknown.

It has been reported that NK cell function is affected by the specific cytokine environment to which it is exposed. During virus infection and tumor progression, TGF- $\beta$ and IL-10 are major inhibitory cytokines, ${ }^{21,22}$ which could induce miR-146a
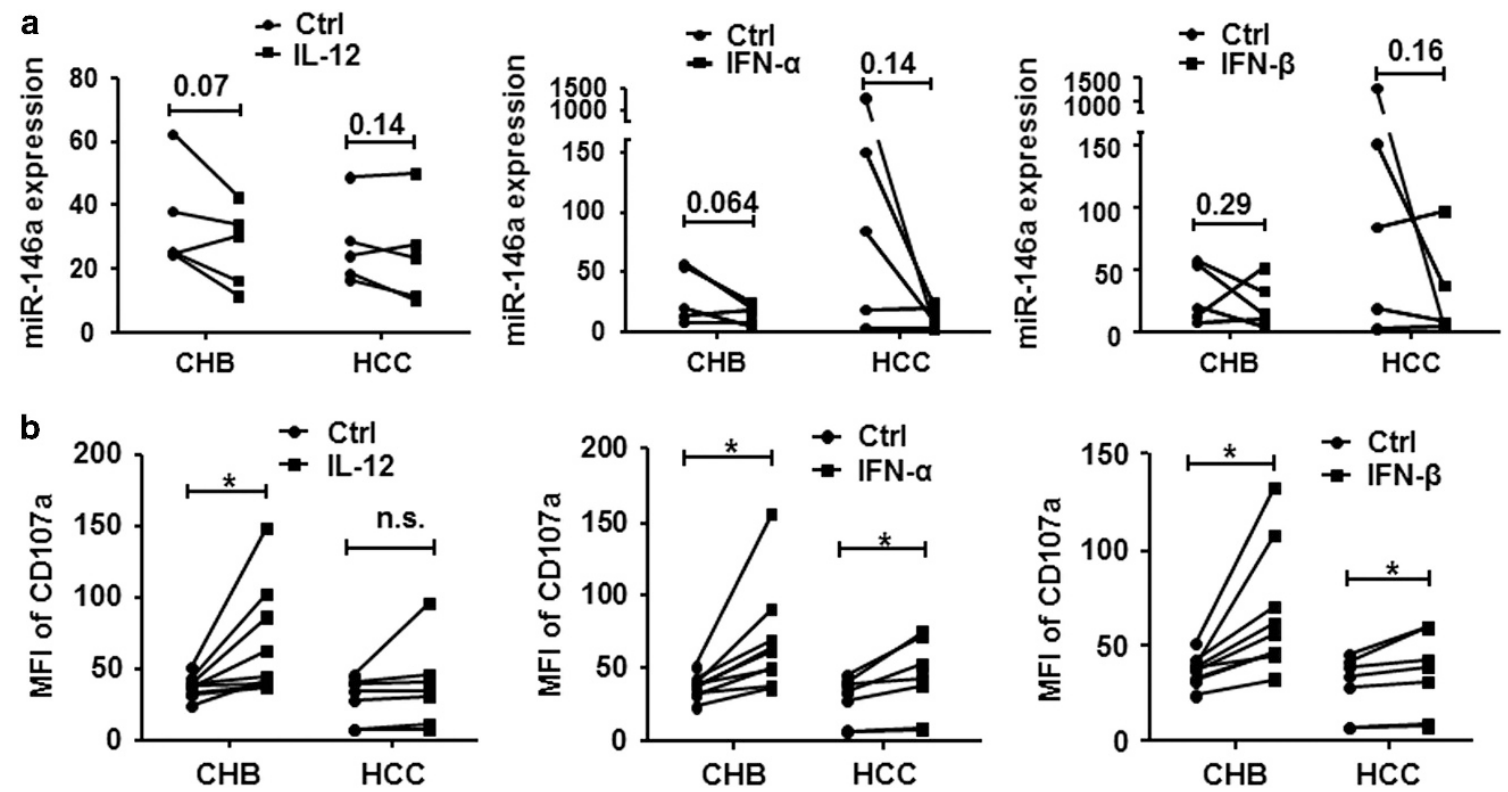

Figure 6 miR-146a expression in NK cells from HCC or CHB patients can be reversed by cytokine-based therapy in vitro. Primary NK cells isolated from CHB and HCC patients were treated with IL-12 (20 ng/ml), IFN- $\alpha(1,000 \mathrm{IU} / \mathrm{ml})$ or IFN- $\beta(1,000 \mathrm{IU} / \mathrm{ml})$ for $12 \mathrm{~h}$. (a) Changes in miR-146a expression levels were detected by qPCR; CHB $(n=5)$ and HCC $(n=5)$. (b) CD107a expression was detected by flow cytometry; $\mathrm{CHB}(n=9)$ and $\mathrm{HCC}(n=7)$. Statistical significance was determined as ${ }^{*} P<0.05$ and $* * P<0.01$ compared with the control. CHB, chronic hepatitis B; HCC, hepatic carcinoma; IL, interleukin; INF, interferon; miR, microRNA; NK, natural killer; qPCR, quantitative PCR. 
expression and result in $\mathrm{NK}$ cell suppression in our study (Figure 3a). In contrast, stimulatory cytokines, including IL-12 and IFN- $\alpha$, as well as the stimulator poly(I:C), downregulated miR-146a expression in NK cells (Figure 3b). In addition to these cytokines, molecules expressed on tumor cells exerted strong stimulatory effects on miR-146a expression in NK cells (Supplementary Figure 2). These data indicate that in HCC or CHB patients, NK cell function can be disturbed by immunosuppressive cytokines and molecules expressed on tumor cells by inducing miRNA-146a expression. However, how these factors regulate the expression of miR-146a is unclear.

A recent paper from our group showed that, in hepatoma cells, miR-146a is regulated by STAT3. ${ }^{30}$ Therefore, we investigated whether STAT3 could also induce miR-146a in NK cells. We found that in NK cells from CHB patients, STAT3 expression was decreased (data not shown), suggesting that miR-146a may not be directly regulated by STAT3 in NK cells. Cameron et al. ${ }^{31}$ have predicted several transcription factor binding sites on the promoter region of miR-146a, including sites for NF-кB, c-Ets and HSF2. We will do further research to explore the associated mechanisms.

miRNAs exert their regulatory effects by targeting specific mRNAs. We submitted molecules important for NK cell activity to bioinformatics analysis using software tools including TargetScan (Whitehead Office of Advancement, Cambridge, MA, USA) and RNAhybrid (Bielefeld Bioinformatics Service, Bielefeld, Germany). TRAIL, ETS-1 and CCR5 were predicted to be potential targets of miR-146a at the posttranscriptional level, but further studies did not suggest they were targets of miR-146a (data not shown). Previous reports have shown that miR-146a regulates Toll-like receptors and RIG-I signaling pathways in monocytes and macrophages by targeting the adapter molecules IRAK1 and TRAF6, ${ }^{19,29}$ and miR-146a is critical for regulatory $\mathrm{T}$-cell-mediated suppressive function and the control of T-helper1 responses by targeting STAT1 in autoimmunity. ${ }^{32}$ Here we found that STAT1, but not IRAK1 and TRAF6, was downregulated at the protein level by miR-146a in NK cells, and NK cells from $\mathrm{CHB}$ and HCC patients also displayed lower STAT1 levels than NK cells from HDs (Figure 4), which could be rescued by overexpression of STAT1 with hsa-STAT1-ORF lentivirus (3'-UTR less; Figure 5). Therefore, STAT1 might be a target of miR-146a for regulating NK cell functions. Significantly, we found that STAT1-activating cytokines type I IFN and IL-12 could downregulate miR-146a levels in NK cells from $\mathrm{CHB}$ and $\mathrm{HCC}$ patients, and augment NK cell cytotoxic activity, suggesting that NK cell function could be partly improved via downregulating miR-146a in cytokine-based immunotherapy. The reason why miR-146a did not target IRAK1 and TRAF6 in NK cells is unclear. It maybe that miRNA 'chooses' different mRNA targets in various types of cells to tune different physiological process.

In conclusion, we found that miR-146a exhibits a negative regulatory effect on NK cell functions by targeting STAT1. Highly expressed miR-146a could be induced by immunosuppressive cytokines, such as IL-10 and TGF- $\beta$, as well as molecules expressed on HCC cells, and subsequently lead to suppression of NK cell function. In contrast, cytokines such as IL-12 and IFN- $\alpha$ could decrease miR-146a expression in NK cells, blocking miR-146a-mediated NK cell impairment and enhancing NK cell cytolysis. These findings provide a novel mechanism involved in NK cell dysfunction and will contribute to the design of effective therapeutic strategies for $\mathrm{CHB}$ and HCC.

\section{CONFLICT OF INTEREST}

The authors declare no conflict of interest.

\section{ACKNOWLEDGEMENTS}

This study was supported by grants from National Natural Science Foundation of China (No. 81172789, 81373222 and 31200651) and National Mega Project on Major Infectious Diseases Prevention and Treatment (No. 2012ZX10002006).

1 Moretta A, Bottino C, Vitale M, Pende D, Cantoni C, Mingari MC et al. Activating receptors and coreceptors involved in human natural killer cell-mediated cytolysis. Annu Rev Immunol 2001; 19: 197-223.

2 Alli RS, Khar A. Interleukin-12 secreted by mature dendritic cells mediates activation of NK cell function. FEBS Lett 2004; 559: 71-76.

3 Biron CA. Interferons alpha and beta as immune regulators-a new look. Immunity 2001; 14: 661-664.

4 Sun C, Fu B, Gao Y, Liao X, Sun R, Tian Z, Wei H. TGF-beta1 down-regulation of NKG2D/DAP10 and 2B4/SAP expression on human NK cells contributes to HBV persistence. PLoS Pathog 2012; 8: e1002594.

5 Fehniger TA, Cai SF, Cao X, Bredemeyer AJ, Presti RM, Ley TJ. Acquisition of murine NK cell cytotoxicity requires the translation of a pre-existing pool of granzyme B and perforin mRNAs. Immunity 2007; 26: 798-811.

6 Bezman NA, Cedars E, Steiner DF, Blelloch R, Hesslein DG, Lanier LL. Distinct requirements of microRNAs in NK cell activation, survival, and function. J Immunol 2010; 185: 3835-3846.

7 Wang P, Gu Y, Zhang Q, Han Y, Hou J, Lin L et al. Identification of resting and type I IFN-activated human NK cell miRNomes reveals microRNA-378 and microRNA-30e as negative regulators of NK cell cytotoxicity. J Immunol 2012; 189: 211-221.

8 Liu X, Wang YL, Sun Q, Yan J, Huang J, Zhu SG et al. Identification of microRNA transcriptome involved in human natural killer cell activation. Immunol Lett 2012; 143: 208-217.

9 Fehniger TA, Wylie T, Germino E, Leong JW, Magrini VJ, Koul S et al. Next-generation sequencing identifies the natural killer cell microRNA transcriptome. Genome Res 2010; 20: 1590-1604.

10 Bezman NA, Chakraborty T, Bender T, Lanier LL. miR-150 regulates the development of NK and iNKT cells. J Exp Med 2011; 208: 2717-2731.

11 Cichocki F, Felices M, McCullar V, Presnell SR, Al-Attar A, Lutz CT et al. Cutting edge: microRNA-181 promotes human NK cell development by regulating Notch signaling. J Immunol 2011; 187: 6171-6175.

12 Sullivan RP, Leong JW, Schneider SE, Keppel CR, French AR, Fehniger TA. MicroRNA-deficient NK cells exhibit decreased survival but enhanced function. J Immunol 2012; 188: 3019-3030.

$13 \mathrm{Ma}$ F, Xu S, Liu X, Zhang Q, Xu X, Liu M et al. The microRNA miR-29 controls innate and adaptive immune responses to intracellular bacterial infection by targeting interferon-gamma. Nat Immunol 2011; 12: 861-869.

14 Trotta R, Chen L, Ciarlariello D, Josyula S, Mao C, Costinean S et al. miR-155 regulates IFN-gamma production in natural killer cells. Blood 2012; 119: 3478-3485. 
$15 \mathrm{Kim}$ TD, Lee SU, Yun S, Sun HN, Lee SH, Kim JW et al. Human microRNA-27a*targets Prf1 and GzmB expression to regulate NK-cell cytotoxicity. Blood 2011; 118: 5476-5486.

16 Gong J, Liu R, Zhuang R, Zhang Y, Fang L, Xu Z et al. miR-30c-1* promotes natural killer cell cytotoxicity against human hepatoma cells by targeting the transcription factor HMBOX1. Cancer Sci 2012; 103: 645-652.

17 Nakasa T, Miyaki S, Okubo A, Hashimoto M, Nishida K, Ochi M et al. Expression of microRNA-146 in rheumatoid arthritis synovial tissue. Arthritis Rheum 2008; 58: 1284-1292.

18 Boldin MP, Taganov KD, Rao DS, Yang LL, Zhao JL, Kalwani M et al. miR-146a is a significant brake on autoimmunity, myeloproliferation, and cancer in mice. J Exp Med 2011; 208: 1189-1201.

19 Hou J, Wang P, Lin L, Liu XG, Ma F, An HZ et al. MicroRNA-146a feedback inhibits RIG-I-dependent type I IFN production in macrophages by targeting TRAF6, IRAK1, and IRAK2. J Immunol 2009; 183: 2150-2158.

20 Wang SF, Zhang XJ, Ju Y, Zhao B, Yan XL, Hu J et al. MicroRNA-146a feedback suppresses $T$ cell immune function by targeting Stat1 in patients with chronic hepatitis B. J Immunol 2013; 191: 293-301.

21 Hanahan D, Weinberg RA. Hallmarks of cancer: the next generation. Cell 2011; 144: 646-674.

22 Li F, Wei H, Wei H, Gao Y, Xu L, Yin W et al. Blocking the natural killer cell inhibitory receptor NKG2A increases activity of human natural killer cells and clears hepatitis B virus infection in mice. Gastroenterology 2013; 144: 392-401.

23 Tang YJ, Luo XB, Cui HJ, Ni XM, Yuan M, Guo YZ et al. MicroRNA-146a contributes to abnormal activation of the type I interferon pathway in human lupus by targeting the key signaling proteins. Arthritis Rheum 2009; 60: 1065-1075.

24 Lee CK, Rao DT, Gertner R, Gimeno R, Frey AB, Levy DE. Distinct requirements for IFNs and STAT1 in NK cell function. J Immunol 2000; 165: 3571-3577.
25 Liang SJ, Wei HM, Sun R, Tian ZG. IFN alpha regulates NK cell cytotoxicity through STAT1 pathway. Cytokine 2003; 23: 190-199.

26 Hauschild A, Gogas H, Tarhini A, Middleton MR, Testori A, Dreno B. Practical guidelines for the management of interferon-alpha-2b side effects in patients receiving adjuvant treatment for melanoma: expert opinion. Cancer 2008; 112: 982-994.

27 Cooksley WG. The role of interferon therapy in hepatitis B. MedGenMed 2004; 6: 16.

28 Ardolino M, Azimi CS, Iannello A, Trevino TN, Horan L, Zhang L et al. Cytokine therapy reverses NK cell anergy in MHC-deficient tumors. J Clin Invest 2014; 124: 4781-4794.

29 Taganov KD, Boldin MP, Chang KJ, Baltimore D. NF-kappa B-dependent induction of microRNA miR-146, an inhibitor targeted to signaling proteins of innate immune responses. Proc Natl Acad Sci USA 2006; 103: 12481-12486.

30 Sun XX, Zhang J, Hou ZH, Han QJ, Zhang C, Tian ZG. miR-146a is directly regulated by STAT3 in human hepatocellular carcinoma cells and involved in anti-tumor immune suppression. Cell Cycle 2015; 14: 243-252.

31 Cameron JE, Yin QY, Fewell C, Lacey M, McBride J, Wang X et al. Epstein-Barr virus latent membrane protein 1 induces cellular MicroRNA miR-146a, a modulator of lymphocyte signaling pathways. J Virol 2008; 82: 1946-1958.

32 Lu LF, Boldin MP, Chaudhry A, Lin LL, Taganov KD, Hanada T. Function of miR-146a in controlling Treg cell-mediated regulation of Th1 responses. Cell 2010; 142: 914-929.

33 Souza-Fonseca-Guimaraes F, Parlato M, Fitting C, Cavaillon JM, Adib-Conquy M. NK cell tolerance to TLR agonists mediated by regulatory T cells after polymicrobial sepsis. J Immunol 2012; 188: 5850-5858.

34 Saez-Borderias A, Romo N, Magri G, Guma M, Angulo A, Lopez-Botet M. IL-12-dependent inducible expression of the CD94/ NKG2A inhibitory receptor regulates CD94/NKG2C(+) NK cell function. J Immunol 2009; 182: 829-836.

Supplementary Information for this article can be found on the Cellular \& Molecular Immunology website (http://www.nature.com/cmi) 